\title{
Long-term structural changes after mTBI, and their relation to post-concussion
}

\section{symptoms}

Philip J. A. Dean ${ }^{1}$, Joao Ricardo Sato ${ }^{2,3}$, Gilson Vieira ${ }^{3}$, Adam McNamara ${ }^{1}$, and Annette

$$
\text { Sterr }^{1}
$$

${ }^{1}$ Department of Psychology, University Of Surrey, Guildford, UK; ${ }^{2}$ Center of Mathematics, Computation and Cognition, Universidade Federal do ABC, São Paulo, Brazil; ${ }^{3}$ NIF/LIM44, Departamento de Radiologia da Faculdade de Medicina da Universidade de São Paulo, São Paulo, Brazil.

Correspondence to: Philip Dean, Department Of Psychology, University Of Surrey, Guildford, Surrey, UK, GU2 7XH

Fax: 01483 682914; Phone: 01483 682877; e-mail: p.dean@surrey.ac.uk 


\begin{abstract}
Primary Objective: To investigate sustained structural changes in the long-term ( $>1$ year) after mild traumatic brain injury (mTBI), and their relationship to ongoing post-concussion syndrome (PCS).

Research Design: Morphological and structural connectivity magnetic resonance imaging (MRI) data were acquired from 16 participants with mTBI and 9 participants without previous head injury.

Main Outcomes and Results: Participants with mTBI had less prefrontal grey matter and lower fractional anisotropy (FA) in the anterior corona radiata and internal capsule. Furthermore, PCS severity was associated with less parietal lobe grey matter and lower FA in the corpus callosum.

Conclusions: There is evidence for both white and grey matter damage in participants with mTBI over a year after injury. Furthermore, these structural changes are greater in those with report more PCS symptoms, suggesting a neurophysiological basis for these persistent symptoms.
\end{abstract}

Keywords: Magnetic Resonance Imaging, Diffusion Tensor Imaging, Cortical Thickness, mild Traumatic Brain Injury, persistent Post-Concussion Syndrome 


\section{Long-term structural changes after $\mathrm{mTBI}$, and their relation to post-concussion}

\section{symptoms}

\section{Introduction}

The majority (70-90\%) of traumatic brain injury (TBI) hospital admissions are classified as mild traumatic brain injury (mTBI) $[1,2]$. However, only an estimated $10-25 \%$ of individuals visiting the emergency department suffering from mTBI are admitted to hospital [3,4] and some do not seek or receive medical attention $[5,6]$. This suggests that incidence of mTBI is much more widespread than the conventional hospital admission figures. In addition, around $5-10 \%$ of individuals who suffer an mTBI go on to have persistent somatic, affective and cognitive symptoms, known as post-concussion syndrome (PCS, [7-10]), which can last a year or more post-injury [11-14].

There is often no visible brain damage after an mTBI when using standard neuroimaging techniques [15-19], leading some to suggest a purely psychogenic origin for persistent PCS [20-23]. However, novel structural imaging acquisition and analysis techniques, such as brain morphometry (e.g. voxel-based morphometry, VBM [24-26]; cortical thickness and volume $[27,28]$ ) and structural connectivity (e.g. diffusion tensor imaging, DTI [26, 29-37]), have shown promise in detecting subtle small-scale damage [18, 38, 39]. Previous studies have reported reduced grey matter in multiple brain regions such as frontal and temporal cortices [24-28], as well as increased mean diffusivity (MD) and reduced fractional anisotropy (FA) in a variety of areas such as the corpus callosum, internal capsule, anterior corona radiata and superior longitudinal fasciculus [26, 30-36]. A smaller number of studies have recorded axial diffusivity (AD) and radial diffusivity (RD), which may provide more detail about axonal and myelin damage respectively [18], and they report reduced AD and RD in similar areas to the FA and MD changes [30, 31, 33, 34]. There is also evidence of an association between greater microstructural damage after brain injury and greater symptom report [26, 34, 37], 
worse cognitive performance [35, 40] and worse outcome [41]. A few studies have investigated the chronic stage after mTBI, and report ongoing structural differences $[24,30$, 34, 41]. However, whilst previous studies have investigated the association of structure with PCS symptom report, only one of these has looked at this association in the chronic stage [34]. This study examined differences in DTI indices at the subacute $(<1$ month) and late $(6$ months) phase post-injury in participants recruited from an emergency department with and without PCS after mTBI. Only one previous study investigated structural changes in the long term (> 1 year) after injury, but did not record PCS symptoms [30].

In addition, previous studies rarely combine morphological and structural connectivity data in the same sample. Whilst structural connectivity, as measured with DTI, has been investigated fairly extensively in participants after mTBI, the vast majority of morphological and volumetric studies have investigated samples with a range of TBI severity, with only a couple specifically examining mTBI $[24,42]$. Yet, morphological and structural connectivity analyses yield complimentary data, with the former usually evaluating changes in grey matter density and thickness and the latter giving a measure of white matter integrity. A combinatory, multimodal approach to investigating structural damage after mTBI may allow a more sensitive detection of subtle deficits, and allow a more individualised profile of damage suited to the heterogeneity of brain injury mechanism in mTBI [18, 38, 39, 43, 44].

This study therefore investigated structural changes in the long term ( $>1$ year post-injury) after mTBI, and the association between these alterations and PCS symptom report employing both morphological and structural connectivity measures in the same sample. It was hypothesised that participants with mTBI would have altered MRI indices, indicating structural damage, and that this damage would be greater in those with greater PCS symptom report. Furthermore, as DTI is a more sensitive measure of microstructural damage [18] than 
cortical thickness or VBM, it would be more likely to find the subtle changes expected after such a mild TBI.

\section{Method}

\section{Participants}

Twenty five participants were included in this study, divided into two groups: 16 participants who suffered an mTBI and 9 participants with no history of brain injury (Control). Post-Concussion symptoms were measured in all participants using the Rivermead Post-Concussion Questionnaire (RPQ) and Rivermead Post-Concussion Questionnaire for Controls (RPQ-C; [13]). The RPQ/RPQ-C scores were used in the subsequent analyses. Group demographics and RPQ scores are shown in table 1.

---Insert table 1 about here---

Recruitment for both participants with mTBI and controls was from a database created by a previous study [45], which was also used in previous cognitive [46] and MRS [47] studies. In brief, this database was created from an online survey designed to access those in the local community who had suffered an mTBI but had not reported to hospital (this could be approx. $75-90 \%$ of those with mTBI $[3,4])$. mTBI diagnosis was according to ICD-10 criteria [10]. Inclusion criteria were that injury occurred at least one year previously. Exclusion criteria were report of litigation, major invasive head injury, chronic pain, or other neurological conditions and visible lesions using standard structural MRI. In addition, measures of depression and anxiety (Hospital Anxiety and Depression Scale, HADS [48]), post-traumatic stress disorder (Impact of Event Scale-Revised: IES-R [49]), and sleep quality (Pittsburgh Sleep Quality Index, PSQI [50]) were acquired, and participants excluded if they met the criteria for clinical symptomatology. The study protocol was given a favourable opinion by 
the University of Surrey Ethics Committee, and informed consent was obtained from every participant.

\section{MRI acquisition}

T1-weighted and Diffusion-Weighted images (DWI) were acquired using a 3T Siemens Trio Scanner (Siemens, Munich, Germany). High-resolution 3D brain MRI images were obtained using a T1-weighted Magnetization Prepared Rapid Acquisition Gradient Echo $($ MPRAGE$)$ pulse sequence $(\mathrm{TR}=1830 \mathrm{~ms} ; \mathrm{TE}=4.43 \mathrm{~ms}$; Inversion Time $=1100 \mathrm{~ms}$; flip angle $=11^{\mathrm{o}} ; \mathrm{FOV}=256 \mathrm{~mm} ; 176$ slices; voxel size $=1 \times 1 \times 1 \mathrm{~mm}^{3} ;$ in-plane matrix $=$ 256x256). DWI data was acquired using a single-shot diffusion-weighted echo-planar imaging sequence, with diffusion gradients applied along 12 directions (b0 $=0$ [1 image], b1 $=1000 \mathrm{smm}^{-2}[12$ images $] ; \mathrm{TR}=8900 \mathrm{~ms}, \mathrm{TE}=100 \mathrm{~ms}$, number of averages $=4,55$ slices,

voxel size $=2.5 \times 2.5 \times 2.5 \mathrm{~mm}^{3}$, in-plane matrix $=88 \times 128$, bandwidth $=2056 \mathrm{~Hz}, \mathrm{FOV}=$ $320 \times 220)$.

\section{Demographic data analysis}

A series of independent t-tests were performed for continuous variables. Differences in gender between groups were investigated using a chi-square test.

\section{Structural MRI Analysis}

Three methods were used to investigate structural changes after mTBI, using T1-weighted images (cortical thickness, voxel-based morphometry) and diffusion weighted images (diffusion tensor imaging).

\section{Cortical thickness analysis}

Cortical thickness was calculated using the FreeSurfer (v. 5.0.0) software package [51, 52]. Pre-processing of the T1-weighted images involved non-uniform intensity correction, affine registration using the Talairach transform to map the images into the Montreal Neurological Institute (MNI) space, intensity normalisation and removal of non-brain tissue. Subsequently, 
grey/white matter and grey matter/CSF boundaries of the cortex were tracked, tessellated and smoothed to produce a surface mesh. Topology correction and surface deformation were then applied. Cortical thickness was calculated as the closest distance from the gray/white boundary to the gray/CSF boundary at each vertex on the tessellated surface [51]. The cortical surface and the subcortical structures in each participant were parcellated into units based on gyral and sulcal landmarks [53].

After pre-processing, cortical geometry was matched across participants by registration of the surface coordinate system from individual cortical folding patterns to a standard spherical atlas [51]. Whole-brain cortical thickness analysis was then performed on each vertex using the general linear model (GLM) embedded in the QDEC (Query, Design, Estimate, Contrast) interface, producing cortical thickness maps illustrating any group differences. Age of participant was included as a covariate to control for the effect of age on brain structure. Cortical thickness in participants who had suffered an mTBI was compared to controls. In addition, correlations between PCS (using RPQ score) and cortical thickness were calculated across participants with mTBI $(n=16)$. Correlation analysis with control participants only yielded no significant results. The results were multiple comparison corrected using False Discovery Rate (FDR) at a threshold of $p<.05$.

\section{Voxel-based morphometry analysis}

Voxel-based morphometry (VBM) analysis was performed using DARTEL [55] in SPM8 (http://www.fil.ion.ucl.ac.uk/spm/). During pre-processing, T1-weighted images were segmented into grey matter, white matter, CSF, skull and other tissue, these segments were aligned across subjects, normalised to MNI space, and smoothed (FHWM $=10 \mathrm{~mm}$ ) and Jacobian scaled.

Second level group analysis was carried out on the grey matter images using a two-sample t-test to look at the effect of mTBI (mTBI vs Control), controlling for age at scan and total 
intra-cranial volume. The interaction between PCS symptom report (as measured using RPQ) and proportion of grey matter was investigated across mTBI participants $(n=16)$ using a regression with RPQ score and age as covariates. The resulting statistical maps were thresholded at $p<.001$, uncorrected. Regression analysis with control participants only yielded no significant results.

\section{Diffusion tensor imaging analysis}

DWI images were motion corrected, realigned to the mean image and normalised to MNI space using the diffusion toolbox in SPM8 (Diffusion II: http://sourceforge.net/projects/spmtools). The gradient information was updated to take into account the realignment and normalisation steps. Diffusion tensor eigenvalues and eigenvectors were calculated to generate the fractional anisotropy (FA) maps for each participant. The FA maps were masked using a whole-brain mask within SPM8 to ensure only brain areas were included in further analysis, and smoothed with a Gaussian kernel of $\mathrm{FHWM}=8 \mathrm{~mm}$.

Second level group analysis was carried out using a two-sample t-test within SPM8 to look at the effect of mTBI (mTBI vs Control), controlling for age at scan. The interaction between PCS symptom report (as measured using RPQ) and FA was investigated across mTBI participants $(\mathrm{n}=16)$ using a regression with RPQ score and age as covariates. The resulting statistical maps were thresholded at $p<.001$, uncorrected. Regression analysis with control participants only yielded no significant results.

\section{Results}

\section{Demographics}

Groups were similar with regards to gender $\left(\chi^{2}(1, \mathrm{~N}=25)=0.001, p=.97\right)$, but participants with mTBI were significantly older (mTBI: 27.0 \pm 1.6 ; Control: $21.9 \pm 1.5 ; t(23)=-2.1, p<$ 
$.05)$ and had greater report of PCS symptoms (mTBI: $18.1 \pm 2.5$; Control: $4.8 \pm 0.9 ; t(19)=-$ $5.0, p<.001)$.

\section{Cortical thickness}

No significant cortical thickness alterations were seen between participants with mTBI and control, and no association was observed between PCS symptom report and cortical thickness in participants with $\mathrm{mTBI}$.

\section{Voxel-based morphometry}

There were modest grey matter differences observed in the voxel based morphometry (VBM) analysis (see figure 1).

Participants with mTBI had a lower proportion of grey matter in small areas within bilateral prefrontal areas (left frontal (cluster size: 143 voxels) and right mid frontal (cluster size: 29 vx) gyri) compared to controls. Greater PCS symptom report across all participants with mTBI was associated with a lower proportion of grey matter in bilateral medial temporal lobe (predominately left [cluster size: $241 \mathrm{vx}$ ], but also right [cluster size: $41 \mathrm{vx}$ ]) as well as left inferior parietal lobe (cluster size: $283 \mathrm{vx}$ ) and right precuneus (cluster size: $519 \mathrm{vx}$ ).

\section{Diffusion tensor imaging}

More substantial white matter differences were observed with diffusion tensor imaging (DTI) analysis (see figure 1). Participants with mTBI revealed reduced fractional anisotropy (FA) in right hemisphere anterior corona radiata and anterior limb internal capsule (combined cluster size: $131 \mathrm{vx}$ ) compared to controls (figure 1). Furthermore, greater PCS symptom report correlated with lower FA in the splenium of the corpus callosum (cluster size: $110 \mathrm{vx}$ ), the fornix (cluster size: $14 \mathrm{vx}$ ) and bilateral frontal medial superior gyrus (cluster size: 206 vx) across all participants with mTBI. 


\section{Discussion}

\section{Principal Findings}

This is the first study to examine indices of sustained structural changes in the long term (> 1 year) after mTBI, and their association with PCS. Importantly, the study also combined different markers of structural integrity, including both morphological (cortical thickness and VBM) and structural connectivity (DTI) analysis in the same sample. As hypothesised, reduced grey and white matter integrity was observed in participants with mTBI compared to controls, and in individuals who reported greater PCS symptoms. In addition, more substantial differences were detected with the structural connectivity (DTI) analysis, which focused on white matter alterations. These results and conclusions will be discussed in detail below.

\section{Grey matter damage}

Reduced prefrontal grey matter concentration has been observed in the acute and chronic stage after mTBI in previous studies [25-28], and the current data presents further evidence of subtle damage to left frontal and right mid frontal gyrus in the long term after injury. An association was also revealed between PCS symptom report and reduced grey matter in the precuneus, medial temporal and inferior parietal lobe. Whilst changes in the temporal and parietal regions (in particular the hippocampus) have been reported by a previous study [27], the study did not test for association with symptom report or behaviour. In fact, only one previous study investigated the association between grey matter concentration and PCS symptom report [26], and found no difference between those with and without PCS. As such, these findings build upon previous research by demonstrating similar grey matter alterations in the long term after injury, and also report a novel finding of an association between grey matter damage and symptom report. Furthermore, the alteration in the precuneus is 
interesting as it is involved in the default mode network (DMN) which has been shown to be altered in connectivity or activation after $\operatorname{mTBI}[56,57]$. Therefore structural differences may underlie the observed functional changes.

\section{White matter damage}

This study found that participants with mTBI exhibited white matter damage in the long term after injury, with reduced FA in white matter tracts in the right frontal lobe (right anterior corona radiata and anterior limb of the internal capsule). Previous DTI studies have also reported altered white matter in these areas at earlier time points after mTBI, with reduced $\mathrm{FA}$ and increased $\mathrm{MD}$ and $\mathrm{AD}$ in anterior corona radiata $[30,33,35]$ and internal capsule $[29,30,32,33]$ and reduced FA in the fornix [58]. This study extends these findings to illustrate that these structural alterations are observable a considerable time ( $>1$ year) after injury.

White matter damage is also commonly reported in the corpus callosum, with reduced FA and increased MD and RD [26-28, 32, 34-37, 58]. Furthermore, greater PCS symptoms have been shown to be associated with reduced FA [37] and increased MD and AD [34] in the corpus callosum. In line with this, this study reported that reduced FA in the splenium of the corpus callosum and fornix was associated with higher PCS symptom report across participants with mTBI, even in the long term after injury. There was also an association between PCS symptom report and damage in white matter areas underlying the frontal cortex (lower FA in frontal medial superior gyrus). Previous studies have observed alteration in these areas after mTBI $[26,59]$, but have also reported an association between alterations in frontal subcortical white matter and PCS symptom report [37].

Taken together, these findings extend the previous research by revealing participants with mTBI exhibit altered white matter in the long term after injury, and that these alterations are 
associated with PCS symptom report. It has been suggested that the microstructural changes observed in the white matter are a likely neuropathological substrate of PCS [37], and the current data support this statement.

\section{Converging evidence}

Overall, the structural imaging data reveal that a number of white matter areas may be compromised even a year or more after injury, with grey matter areas affected to a lesser degree. In fact, some of the grey matter areas (right prefrontal) may be related to underlying white matter damage (right anterior corona radiata and internal capsule). PCS was associated with indicators of damage to both grey and white matter, suggesting that this changes may underlie at least some of the symptoms reported. These data were acquired in participants who were not recruited from hospitals, and so may represent the milder end of the spectrum of mTBI, and yet still presented with observable structural changes in the long-term after injury. As younger age groups are more likely to have these persistent symptoms [60], this is a potentially long-lasting impairment in the early stages of life. If there are long-term structural alterations after mTBI, and they are related to persistent symptoms, this will have clinical implications, with longer-term follow-up and care required. Relating the persistent symptoms to an underlying structural change may enable more specifically tailored therapeutic intervention. Furthermore, this study suggests that structural alterations are more likely to be found using structural connectivity measures such as DTI, thus enabling better diagnosis and follow-up.

In both the VBM and DTI analyses the areas associated with PCS symptom report were distinct from those that differed between participants with mTBI and non-head injured controls. This suggests that the heterogeneity of injury after mTBI may be masking 
underlying structural changes, and using behavioural data or PCS symptoms as covariates may be one way to control for the heterogeneity.

Recent review articles have advocated another approach, forgoing group-level analysis for a more individualised method [18, 39]. The group-level analysis assumes that heterogenous injuries results in damage to homogenous areas of the brain. More sensitive measures of fine scale damage can be achieved detecting clusters of altered FA for each individual compared to a control cohort, and then for instance counting the number of clusters as a measure of lesion load [61-63]. A more individualised analysis approach such as this would only be possible in future studies with a larger cohort.

\section{Comparison of methodologies}

As hypothesised, more substantial structural differences were detected with the structural connectivity (DTI) analysis, compared to morphological and volumetric analysis. Indeed, one of the morphological analysis techniques (cortical thickness) yielded no significant results. It would therefore seem that DTI is the most sensitive method for detection of alteration after mTBI [18]. DTI can record microstructural changes in tissue integrity, unlike conventional MRI [18], and some believe it may enable individual level diagnosis of mTBI [39]. Furthermore, there is some convergence and consistency in the DTI measures acquired across different studies after mTBI $[18,39,44]$, whereas volumetric analysis has a more complex association with outcome, and varies with age and the nature, severity, location and mechanism of injury [39].

However, these methods also measure different aspects of brain structure which may be altered to a different extent by mild TBI. Axonal fibre stretching and tearing, causing diffuse axonal injury (DAI), is thought to be a primary mechanism of injury in mild TBI, with grey matter atrophy secondary to this, and more likely in more severe TBI. Grey matter damage 
caused by brain-bone impact may be less common in mild TBI, and would certainly be more variable in its location than the forces that stretch and tear white matter tracts. If this is the case, larger-scale and more consistent white matter damage after mTBI, with comparatively less grey matter atrophy, is not unexpected. In a diagnostic setting this dissociation is not as important, and structural connectivity measures will be more likely to distinguish between injury groups no matter what the underlying cause. However, in a research and treatment setting, a complete picture of the damage is only possible using a multi-modal approach [18]. The mechanism and severity of injury may influence whether there are greater grey matter alterations or white matter changes, and the techniques to examine these aspects are complimentary.

The data presented here are from a relatively small sample of participants, in a crosssectional design. This leads to certain caveats when interpreting the data. Firstly, a small sample size could lead to low statistical power, and this study could be interpreted as revealing that cortical thickness and VBM are more affected by low statistical power. If this were the case, it would also suggest that DTI is more suited to an individualised analysis such as a clinical setting. Replication of this study in a larger sample size could help corroborate the findings of structural alterations in the long-term after injury and would also allow a more comprehensive examination of the various imaging methods. However, the areas affected in this study are similar to those seen in previous studies for both grey and white matter injuries after injury. In particular the corpus callosum and the medial temporal lobes are thought to be especially vulnerable areas after mTBI [64]. These results are in accordance with the previous literature and are therefore valid and of interest to the wider brain injury community.

Secondly, the nature of our sample, which looked at individuals who suffered an mTBI but received no hospital attention at time of injury, necessitated a cross-sectional design for the study. There could be no initial pre-injury assessment and no immediate follow-up after 
injury and during recovery, as the injury was never reported to hospital. The reason for sampling this population was that it is estimated that the majority of individuals do not report to hospital after injury $[3,4,5,6]$, and these individuals would likely represent the milder end of the mTBI spectrum. In addition, they represent an under-researched population within mTBI. However, using a cross-sectional approach as opposed to a longitudinal approach (recommended for a clearer picture of damage after injury [65]) means that it is difficult to infer a definitive causal link between the initial injury and the symptoms and structural alterations observed. Other co-morbidites such as age, depression or substance abuse could be linked to the structural alterations observed. In this study these co-variables were recorded and screened appropriately in order to reduce their effect on the data, and clarify interpretation.

\section{Conclusion}

This study reports that participants with mTBI exhibit structural alterations even a year or more after injury, and even in a sample who did not report to hospital with the injury. Furthermore, these alterations are associated with ongoing PCS, suggesting a neurophysiological basis for these persistent symptoms. Structural connectivity measures seem to be more sensitive to damage after mTBI, although this could also be due to the type of injury sustained in such mild TBI. Future analyses would benefit from multi-modal neuroimaging data, and a more individually tailored analysis. 


\section{Informed Consent Statement}

All procedures were in accordance with the ethical standards of the responsible committee on human experimentation (institutional and national) and with the Helsinki Declaration of 1975, as revised in 2000. The study protocol was given a favourable opinion by the University of Surrey Ethics Committee, and informed consent was obtained from all participants.

\section{Declaration of Interest}

This research was supported by a Wingate Foundation Scholarship, UK. 


\section{References}

1. Cassidy JD, Carroll LJ, Peloso PM, Borg J, von Holst H, Holm L, Kraus J, Coronado VG. Incidence, risk factors and prevention of mild traumatic brain injury: results of the WHO Collaborating Centre Task Force on Mild Traumatic Brain Injury. J Rehabil Med 2004(43 Suppl):28-60.

2. Goodacre S. Hospital admissions with head injury following publication of NICE guidance. Emerg Med J 2008;25(9):556-7.

3. Bazarian JJ, McClung J, Shah MN, Cheng YT, Flesher W, Kraus J. Mild traumatic brain injury in the United States, 1998--2000. Brain Inj 2005;19(2):85-91.

4. Sosin DM, Sniezek JE, Thurman DJ. Incidence of mild and moderate brain injury in the United States, 1991. Brain Inj 1996;10(1):47-54.

5. Langlois JA, Rutland-Brown W, Wald MM. The epidemiology and impact of traumatic brain injury: a brief overview. J Head Trauma Rehabil 2006;21(5):375-8.

6. NCIPC. Report to Congress on Mild Traumatic Brain Injury in the United States: Steps to Prevent a Serious Public Health Problem. . Atlanta, GA: Centers for Disease Control and Prevention; 2003.

7. Bigler ED. Neuropsychology and clinical neuroscience of persistent post-concussive syndrome. J Int Neuropsychol Soc 2008;14(1):1-22.

8. Iverson GL. Outcome from mild traumatic brain injury. Curr Opin Psychiatry 2005;18(3):301-17.

9. Ryan LM, Warden DL. Post concussion syndrome. Int Rev Psychiatry 2003;15(4):310-6.

10. WHO. International Statistical Classification of Diseases and Related Health Problems. Geneva, Switzerland: World Health Organisation; 1992. 
11. Dikmen S, Machamer J, Fann JR, Temkin NR. Rates of symptom reporting following traumatic brain injury. J Int Neuropsychol Soc 2010;16(3):401-11.

12. Hessen E, Anderson V, Nestvold K. MMPI-2 profiles 23 years after paediatric mild traumatic brain injury. Brain Inj 2008;22(1):39-50.

13. Sterr A, Herron K, Hayward C, Montaldi D. Are mild head injuries as mild as we think? Neurobehavioral concomitants of chronic post-concussion syndrome. BMC Neurol 2006;6(1):7.

14. Stulemeijer M, Vos PE, Bleijenberg G, van der Werf SP. Cognitive complaints after mild traumatic brain injury: things are not always what they seem. J Psychosom Res 2007;63(6):637-45.

15. Belanger HG, Vanderploeg RD, Curtiss G, Warden DL. Recent neuroimaging techniques in mild traumatic brain injury. J Neuropsychiatry Clin Neurosci 2007;19(1):5-20.

16. Lee H, Wintermark M, Gean AD, Ghajar J, Manley GT, Mukherjee P. Focal lesions in acute mild traumatic brain injury and neurocognitive outcome: CT versus 3T MRI. J Neurotrauma 2008;25(9):1049-56.

17. Lewine JD, Davis JT, Bigler ED, Thoma R, Hill D, Funke M, Sloan JH, Hall S, Orrison WW. Objective documentation of traumatic brain injury subsequent to mild head trauma: multimodal brain imaging with MEG, SPECT, and MRI. J Head Trauma Rehabil 2007;22(3):141-55.

18. Shenton ME, Hamoda HM, Schneiderman JS, Bouix S, Pasternak O, Rathi Y, Vu MA, Purohit MP, Helmer K, Koerte I and others. A review of magnetic resonance imaging and diffusion tensor imaging findings in mild traumatic brain injury. Brain Imaging Behav 2012. 
19. Topal NB, Hakyemez B, Erdogan C, Bulut M, Koksal O, Akkose S, Dogan S, Parlak M, Ozguc H, Korfali E. MR imaging in the detection of diffuse axonal injury with mild traumatic brain injury. Neurol Res 2008;30(9):974-8.

20. Bailey CM, Echemendia RJ, Arnett PA. The impact of motivation on neuropsychological performance in sports-related mild traumatic brain injury. J Int Neuropsychol Soc 2006;12(4):475-84.

21. Belanger HG, Spiegel E, Vanderploeg RD. Neuropsychological performance following a history of multiple self-reported concussions: a meta-analysis. J Int Neuropsychol Soc 2010;16(2):262-7.

22. Mittenberg W, DiGiulio DV, Perrin S, Bass AE. Symptoms following mild head injury: expectation as aetiology. J Neurol Neurosurg Psychiatry 1992;55(3):200-4.

23. Mulhern S, McMillan TM. Knowledge and expectation of postconcussion symptoms in the general population. J Psychosom Res 2006;61(4):439-45.

24. Cohen BA, Inglese M, Rusinek H, Babb JS, Grossman RI, Gonen O. Proton MR spectroscopy and MRI-volumetry in mild traumatic brain injury. AJNR Am J Neuroradiol 2007;28(5):907-13.

25. Gale SD, Baxter L, Roundy N, Johnson SC. Traumatic brain injury and grey matter concentration: a preliminary voxel based morphometry study. J Neurol Neurosurg Psychiatry 2005;76(7):984-8.

26. Messe A, Caplain S, Paradot G, Garrigue D, Mineo JF, Soto Ares G, Ducreux D, Vignaud F, Rozec G, Desal H and others. Diffusion tensor imaging and white matter lesions at the subacute stage in mild traumatic brain injury with persistent neurobehavioral impairment. Hum Brain Mapp 2010.

27. Warner MA, Marquez de la Plata C, Spence J, Wang JY, Harper C, Moore C, Devous M, Diaz-Arrastia R. Assessing spatial relationships between axonal integrity, regional 
brain volumes, and neuropsychological outcomes after traumatic axonal injury. $\mathbf{J}$ Neurotrauma 2010;27(12):2121-30.

28. Yurgelun-Todd DA, Bueler CE, McGlade EC, Churchwell JC, Brenner LA, LopezLarson MP. Neuroimaging correlates of traumatic brain injury and suicidal behavior. J Head Trauma Rehabil 2011;26(4):276-89.

29. Cubon VA, Putukian M, Boyer C, Dettwiler A. A diffusion tensor imaging study on the white matter skeleton in individuals with sports-related concussion. $\mathrm{J}$ Neurotrauma 2011;28(2):189-201.

30. Kasahara K, Hashimoto K, Abo M, Senoo A. Voxel- and atlas-based analysis of diffusion tensor imaging may reveal focal axonal injuries in mild traumatic brain injury - comparison with diffuse axonal injury. Magn Reson Imaging 2012.

31. Kraus MF, Susmaras T, Caughlin BP, Walker CJ, Sweeney JA, Little DM. White matter integrity and cognition in chronic traumatic brain injury: a diffusion tensor imaging study. Brain 2007;130(Pt 10):2508-19.

32. Lipton ML, Gellella E, Lo C, Gold T, Ardekani BA, Shifteh K, Bello JA, Branch CA. Multifocal white matter ultrastructural abnormalities in mild traumatic brain injury with cognitive disability: a voxel-wise analysis of diffusion tensor imaging. $\mathbf{J}$ Neurotrauma 2008;25(11):1335-42.

33. Mayer AR, Ling J, Mannell MV, Gasparovic C, Phillips JP, Doezema D, Reichard R, Yeo RA. A prospective diffusion tensor imaging study in mild traumatic brain injury. Neurology 2010;74(8):643-50.

34. Messe A, Caplain S, Pelegrini-Issac M, Blancho S, Montreuil M, Levy R, Lehericy S, Benali H. Structural integrity and postconcussion syndrome in mild traumatic brain injury patients. Brain Imaging Behav 2012;6(2):283-92. 
35. Niogi SN, Mukherjee P, Ghajar J, Johnson C, Kolster RA, Sarkar R, Lee H, Meeker M, Zimmerman RD, Manley GT and others. Extent of microstructural white matter injury in postconcussive syndrome correlates with impaired cognitive reaction time: a 3T diffusion tensor imaging study of mild traumatic brain injury. AJNR Am J Neuroradiol 2008;29(5):967-73.

36. Rutgers DR, Toulgoat F, Cazejust J, Fillard P, Lasjaunias P, Ducreux D. White matter abnormalities in mild traumatic brain injury: a diffusion tensor imaging study. AJNR Am J Neuroradiol 2008;29(3):514-9.

37. Smits M, Houston GC, Dippel DW, Wielopolski PA, Vernooij MW, Koudstaal PJ, Hunink MG, van der Lugt A. Microstructural brain injury in post-concussion syndrome after minor head injury. Neuroradiology 2011;53(8):553-63.

38. Gonzalez PG, Walker MT. Imaging modalities in mild traumatic brain injury and sports concussion. PM R 2011;3(10 Suppl 2):S413-24.

39. Hunter JV, Wilde EA, Tong KA, Holshouser BA. Emerging imaging tools for use with traumatic brain injury research. J Neurotrauma 2012;29(4):654-71.

40. Wu TC, Wilde EA, Bigler ED, Yallampalli R, McCauley SR, Troyanskaya M, Chu Z, Li X, Hanten G, Hunter JV and others. Evaluating the relationship between memory functioning and cingulum bundles in acute mild traumatic brain injury using diffusion tensor imaging. J Neurotrauma 2010;27(2):303-7.

41. Newcombe V, Chatfield D, Outtrim J, Vowler S, Manktelow A, Cross J, Scoffings D, Coleman M, Hutchinson P, Coles J and others. Mapping traumatic axonal injury using diffusion tensor imaging: correlations with functional outcome. PLoS One 2011;6(5):e19214.

42. Holli KK, Waljas M, Harrison L, Liimatainen S, Luukkaala T, Ryymin P, Eskola H, Soimakallio S, Ohman J, Dastidar P. Mild Traumatic Brain Injury Tissue Texture 
Analysis Correlated to Neuropsychological and DTI Findings. Acad Radiol 2010;17(9):1096-1102.

43. Irimia A, Chambers MC, Torgerson CM, Filippou M, Hovda DA, Alger JR, Gerig G, Toga AW, Vespa PM, Kikinis R and others. Patient-tailored connectomics visualization for the assessment of white matter atrophy in traumatic brain injury. Front Neurol 2012;3:10.

44. Slobounov S, Gay M, Johnson B, Zhang K. Concussion in athletics: ongoing clinical and brain imaging research controversies. Brain Imaging Behav 2012;6(2):224-43.

45. Dean PJ, O'Neill D, Sterr A. Post-concussion syndrome: prevalence after mild traumatic brain injury in comparison with a sample without head injury. Brain Inj 2012;26(1):14-26.

46. Dean PJ, Sterr A. Long-term effects of mild traumatic brain injury on cognitive performance. Front Hum Neurosci 2013;7:30.

47. Dean PJ, Otaduy MC, Harris LM, McNamara A, Seiss E, Sterr A. Monitoring longterm effects of mild traumatic brain injury with magnetic resonance spectroscopy: a pilot study. Neuroreport 2013;24(12):677-81.

48. Zigmond AS, Snaith RP. The hospital anxiety and depression scale. Acta Psychiatr Scand 1983;67(6):361-70.

49. Weiss DS. The Impact of Event Scale: Revised In: Wilson JPaT, C.S., editor. CrossCultural Assessment of Psychological Trauma and PTSD: Springer US; 2007. p 218-.

50. Buysse DJ, Reynolds CF, 3rd, Monk TH, Berman SR, Kupfer DJ. The Pittsburgh Sleep Quality Index: a new instrument for psychiatric practice and research. Psychiatry Res 1989;28(2):193-213.

51. Fischl B, Dale AM. Measuring the thickness of the human cerebral cortex from magnetic resonance images. Proc Natl Acad Sci U S A 2000;97(20):11050-5. 
52. Dale AM, Fischl B, Sereno MI. Cortical surface-based analysis. I. Segmentation and surface reconstruction. Neuroimage 1999;9(2):179-94.

53. Desikan RS, Segonne F, Fischl B, Quinn BT, Dickerson BC, Blacker D, Buckner RL, Dale AM, Maguire RP, Hyman BT and others. An automated labeling system for subdividing the human cerebral cortex on MRI scans into gyral based regions of interest. Neuroimage 2006;31(3):968-80.

54. Fischl B, Sereno MI, Dale AM. Cortical surface-based analysis. II: Inflation, flattening, and a surface-based coordinate system. Neuroimage 1999;9(2):195-207.

55. Shen S, Sterr A. Is DARTEL-based voxel-based morphometry affected by width of smoothing kernel and group size? A study using simulated atrophy. J Magn Reson Imaging 2013;37(6):1468-75.

56. Mayer AR, Mannell MV, Ling J, Gasparovic C, Yeo RA. Functional connectivity in mild traumatic brain injury. Hum Brain Mapp 2011.

57. Zhang K, Johnson B, Gay M, Horovitz SG, Hallett M, Sebastianelli W, Slobounov S. Default mode network in concussed individuals in response to the YMCA physical stress test. J Neurotrauma 2012;29(5):756-65.

58. Singh M, Jeong J, Hwang D, Sungkarat W, Gruen P. Novel diffusion tensor imaging methodology to detect and quantify injured regions and affected brain pathways in traumatic brain injury. Magn Reson Imaging 2010;28(1):22-40.

59. Salmond CH, Menon DK, Chatfield DA, Williams GB, Pena A, Sahakian BJ, Pickard JD. Diffusion tensor imaging in chronic head injury survivors: correlations with learning and memory indices. Neuroimage 2006;29(1):117-24.

60. Rosenbaum SB, Lipton ML. Embracing chaos: the scope and importance of clinical and pathological heterogeneity in mTBI. Brain Imaging Behav 2012;6(2):255-82. 
61. Ling JM, Pena A, Yeo RA, Merideth FL, Klimaj S, Gasparovic C, Mayer AR. Biomarkers of increased diffusion anisotropy in semi-acute mild traumatic brain injury: a longitudinal perspective. Brain 2012;135(Pt 4):1281-92.

62. Jorge RE, Acion L, White T, Tordesillas-Gutierrez D, Pierson R, Crespo-Facorro B, Magnotta VA. White matter abnormalities in veterans with mild traumatic brain injury. Am J Psychiatry 2012;169(12):1284-91.

63. Davenport ND, Lim KO, Armstrong MT, Sponheim SR. Diffuse and spatially variable white matter disruptions are associated with blast-related mild traumatic brain injury. Neuroimage 2012;59(3):2017-24.

64. Bigler ED, Maxwell WL. Neuropathology of mild traumatic brain injury: relationship to neuroimaging findings. Brain Imaging Behav 2012;6(2):108-36.

65. Ross, DE. Review of longitudinal studies of MRI brain volumetry in patients with traumatic brain injury. Brain Injury 2011; 25(13-14): 1271-1278 
Table 1. Participant information.

RPQ: Rivermead Post-Concussion Questionnaire sum score.

\begin{tabular}{|c|c|c|c|c|}
\hline Group & Age & Gender & RPQ & Cause Of Injury \\
\hline mTBI & 24 & F & 35 & Motor vehicle accident \\
\hline & 19 & M & 31 & Sport concussion \\
\hline & 26 & F & 29 & Accidental fall \\
\hline & 23 & F & 25 & Motor vehicle accident \\
\hline & 21 & F & 23 & Hit head upon object \\
\hline & 37 & F & 23 & Accidental fall \\
\hline & 36 & F & 21 & Hit head upon object \\
\hline & 19 & M & 15 & Accidental fall \\
\hline mTBI & 23 & F & 18 & Hit head upon object \\
\hline & 29 & M & 17 & Accidental fall \\
\hline & 25 & F & 16 & Hit head upon object \\
\hline & 26 & F & 16 & Hit head upon object \\
\hline & 22 & M & 12 & Sport concussion \\
\hline & 33 & M & 6 & Sport concussion \\
\hline & 29 & M & 2 & Accidental fall \\
\hline & 39 & M & 0 & Hit head upon object \\
\hline Control & 23 & F & 8 & \\
\hline & 25 & F & 8 & \\
\hline & 18 & M & 7 & \\
\hline & 19 & M & 6 & \\
\hline & 20 & F & 5 & \\
\hline & 18 & F & 3 & \\
\hline & 20 & M & 3 & \\
\hline & 22 & M & 3 & \\
\hline & 32 & F & 0 & \\
\hline
\end{tabular}




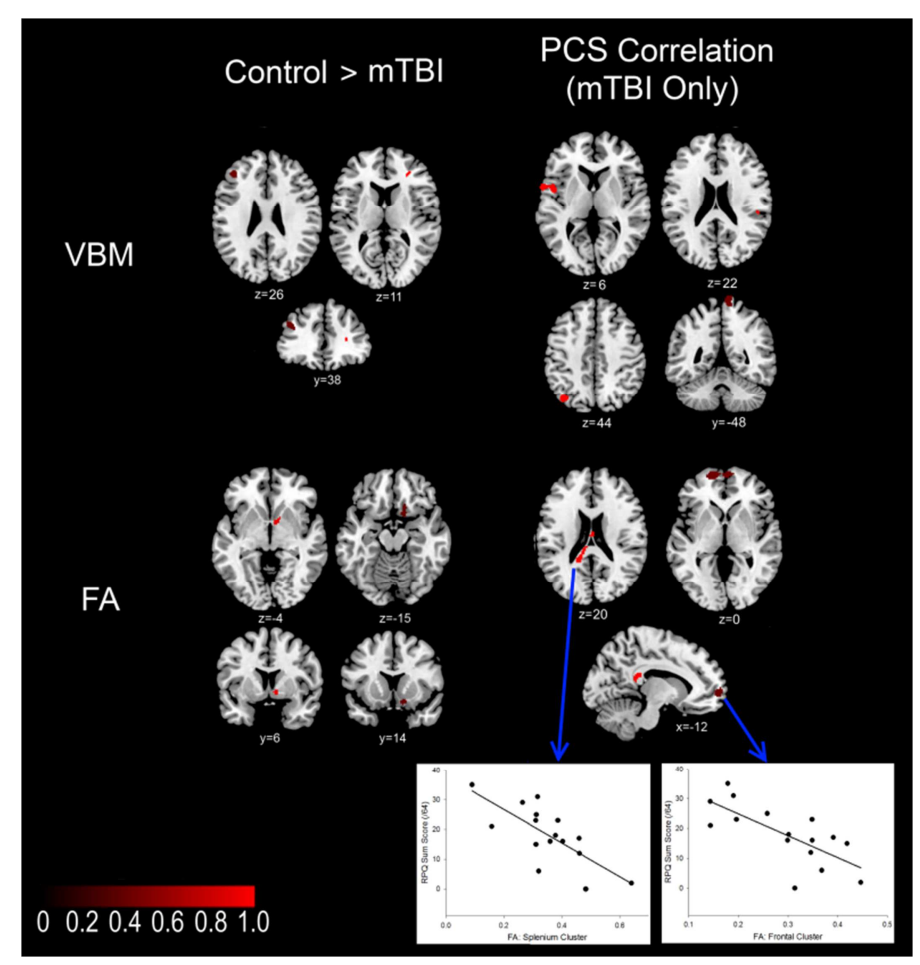

Fig. 1 Illustration differences in VBM and DTI indices between groups (mTBI/Control) and correlation with PCS symptom report (using RPQ sum score). PCS correlation is within participants with mTBI only. Row 1: Voxel-based morphometry (VBM), row 2: DTI: fractional anisotropy (FA), with scatter plot for the interaction between PCS symptom report and FA in the splenium of the corpus callosum. Location of brain section is reported underneath each illustration. Threshold shown: $\mathrm{p}<0.001$, uncorrected. 